\title{
Radical Ring-Opening of Oxetanes Enabled by Co-Catalysis
}

\author{
Aleksandra Potrząsaj, ${ }^{\ddagger}$ Michał Ociepa, ${ }^{\ddagger}$ Wojciech Chaładaj, ${ }^{*}$ Dorota Gryko* \\ Institute of Organic Chemistry Polish Academy of Sciences, Kasprzaka 44/52, 01-224 Warsaw, Poland \\ Supporting Information Placeholder
}

\begin{abstract}
Oxetanes are valuable building blocks due to their well-explored propensity to undergo ringopening reactions with diverse nucleophiles. However, their application as precursors of radical species remains unexplored. Herein, we present a cobalt catalysis-based strategy to access various modes of radical reactivity via oxetane ring opening. The developed method involves formation of an alkylated Co-complex intermediate from vitamin $\mathrm{B}_{12}$ and oxetane. Homolytic cleavage of the $\mathrm{Co}-\mathrm{C}$ bond generates nucleophilic radicals that engage in reactions with SOMOphiles and low-valent transition metals. The scope of the developed reactions is broad with various functional groups being well tolerated. Importantly, the regioselectivity of these processes complements known methodologies.
\end{abstract}

\section{INTRODUCTION}

The oxetane moiety is present in many natural compounds and drug molecules. Due to their position as both carbonyl and gem-dimethyl group surrogates, oxetanes are important scaffolds in drug discovery (bioisosters). They are also valuable as C-3 building blocks for the synthesis of highly functionalized organic frameworks. ${ }^{1}$

The high ring-strain governs the reactivity of oxetanes facilitating a plethora of transformations, among which, strategies based on breaking the $\mathrm{C}-\mathrm{O}$ or $\mathrm{C}-\mathrm{C}$ bonds predominate. ${ }^{2}$ Indeed, the most explored reaction is nucleophilic ring-opening with heteroatom nucleophiles but there are also few reports describing their reactions with C-nucleophiles., ${ }^{1,3,4}$ For example, desymmetrization of 3substituted oxetanes has proved to be a powerful strategy for constructing chiral alcohols or enlarged heterocycles. ${ }^{2,5-7}$ These methods rely on transition-metal catalysis, organocatalysis, or employing enzymes. On the other hand, the less explored $\mathrm{C}-\mathrm{C}$ bond modifications often involve rearrangements, $\beta$-eliminations, or ring expansions.

In addition, oxetanes are a convenient source of $\alpha$-oxy radicals. Recently, the MacMillan group developed an efficient methodology for the deoxygenative arylation of alcohols and for the $\alpha$-arylation of ethers, including oxetanes ${ }^{8}{ }^{8}$ while Ravelli et al. demonstrated their photochemical reaction with electron-deficient olefins. ${ }^{9}$ These examples represent radical functionalizations, where the 4-membered ring is preserved.

On the other hand, despite significant strain energy of the oxetanes (c.a. $106 \mathrm{~kJ} / \mathrm{mol}$ ), their application in radical transformations initiated by opening of strained-ring systems is limited to only few examples. In 2008, Grimme and Gansäuer developed a $\mathrm{Cp}_{2}$ TiCl-catalyzed system for the generation of $\gamma$-titanoxy radicals (Scheme 1). ${ }^{10}$ These behave like typical alkyl radicals and undergo reductive ring opening, dimerization, or react with external SOMOphiles (significant excess, 10 equiv, of a trapping reagent is required). The authors however concluded that ' $\gamma$-titanoxy radicals are not suitable for efficient formation of C-C bonds.' 


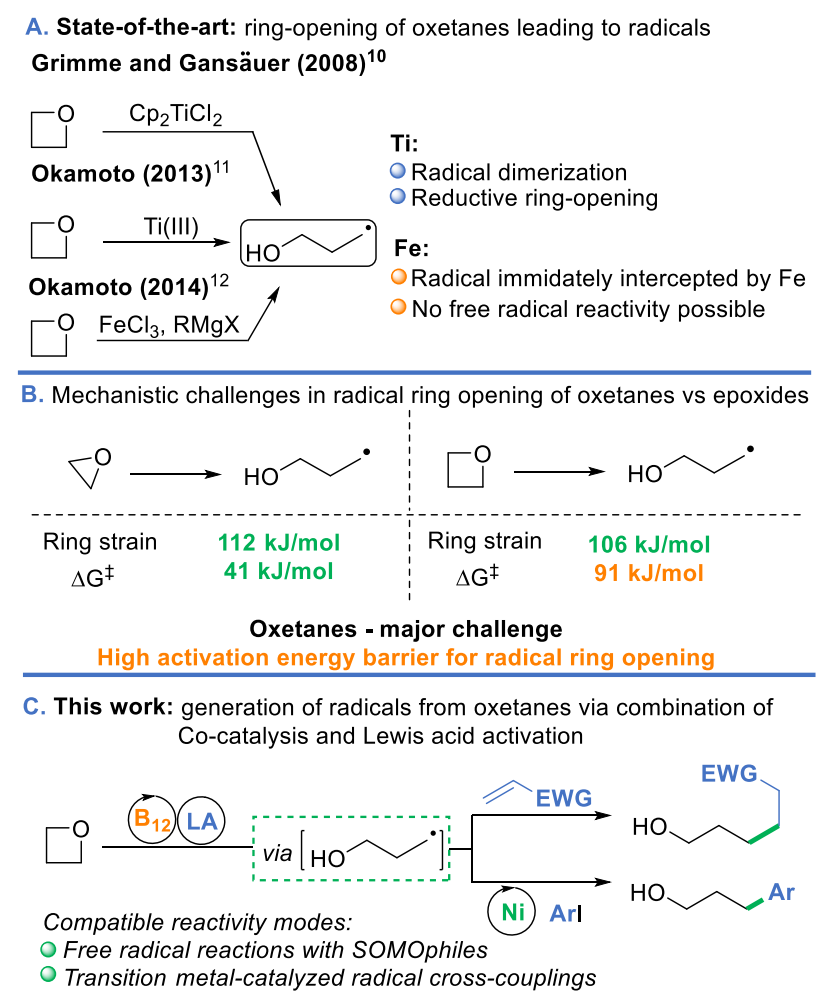

Similar reactivity was achieved by the Okamoto group in the presence of low-valent titanium alkoxides. ${ }^{11}$ The same group also used iron-catalysis to access 3-oxidopropylmagnesium compounds from 2-substituted oxetanes. ${ }^{12}$ Although, the above transformation likely proceeds via an $\gamma$-oxidoradical intermediate, it is immediately intercepted by an iron catalyst, which precludes free-radical reactivity. Despite the immense importance of these seminal contributions, the possibility to access various modes of radical reactivity via ring opening of oxetanes remains challenging.

Recently, we have reported a polarity reversal strategy enabling functionalization of strained cycloalkanes ${ }^{13}$ and regioselective ring-opening of epoxides (oxiranes). ${ }^{14}$ The crucial step of these processes involve the formation of alkyl cobalamins from vitamin $\mathrm{B}_{12}$ and electrophilic substrates followed by the homolytic cleavage of the Co-C leading to alkyl radicals. Although the strain energies of oxirane $(112 \mathrm{kcal} / \mathrm{mol})$ and oxetane $(106 \mathrm{kcal} / \mathrm{mol})$ rings are on a similar level, ring opening of the latter is kinetically unfavorable due to the high activation energy of this process (Scheme 1B). We envisioned that the combination of our cobalt-catalyzed strategy and a suitable oxetane activation method will enable the generation of alkyl radicals from oxetanes, by overcoming the challenging kinetics of the ring opening step (Scheme 1C).

Herein, we report a general method for the generation of nucleophilic C-centered radicals from oxetanes in the vitamin $\mathrm{B}_{12}$-catalyzed oxetane ring-opening reaction and its application in both $\mathrm{Ni}$ catalyzed cross-electrophile coupling and the Giese-type addition.

\section{RESULTS AND DISCUSSION}

Nucleophilic radicals engage in cross-electrophile coupling with aryl halides via cooperative $\mathrm{Co} / \mathrm{Ni}$ catalysis. According to the literature, the interception of alkyl radicals generated in the Co-catalytic cycle with $\mathrm{Ni}$-species, either before or after the oxidative addition, leads to the formation of a C-C bond. ${ }^{15-21}$ We hypothesize that radicals formed from oxetanes would follow this pathway. To this end, our experimental investigations began with the conditions adopted from those developed for epoxides. ${ }^{14}$ 
Unsurprisingly, the model reaction of 3-phenyloxetane (1) with aryl iodide $\mathbf{2}$ without any activator did not lead to desired product $4 \mathbf{a}$ (Scheme 2).

Scheme 2. Co-catalyzed functionalization of oxetanes involving ring-opening

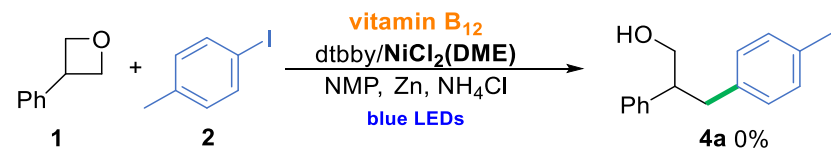

DFT calculations show that the free Gibbs energy for the ring opening of oxetanes with the model corrin is substantially higher than the one calculated for the generation of radicals from epoxides (Scheme 3). All the calculations were performed with the Gaussian 16 package. ${ }^{22}$ Geometry optimizations were computed at BP86/6-31G(d) level of theory with the D3 version of Grimme's empirical dispersion correction ${ }^{23}$ and SMD model of solvation $(\mathrm{MeCN}) .{ }^{24}$ In this process, the Co-corrin complex, bearing 15 methyl groups, reflects the substitution pattern at the rim of the approximated structure of heptamethyl cobyrinate.

Due to the Lewis basicity of the oxygen atom, oxetanes can be activated by acids. ${ }^{1,3}$ Furthermore, they also influence the regioselectivity of ring opening reactions. ${ }^{1,3}$ The use of these reagents, from the standpoint of our catalytic approach, poses several challenges. We expected that hydrophilic vitamin $\mathrm{B}_{12}$ bearing Lewis basic amide groups may undergo side-reactions with acids, hindering its catalcatalytic activity. Moreover, the activated oxonium cation may be prone to reductive cleavage of the C-O bond. With this in mind, hydrophobic heptamethyl cobyrinate (3) in combination with a broad variety of acid additives was tested in the model reaction (Scheme 4, for more details see SI).

Scheme 3. Gibbs free energy barriers for the opening of epoxides and oxetanes with the Co(I)-corrin complex calculated at PB86-D3/6-311++G(2df,p)/SMD (MeCN)//BP86-D3/6-31G(d)/SMD (MeCN) level of theory.

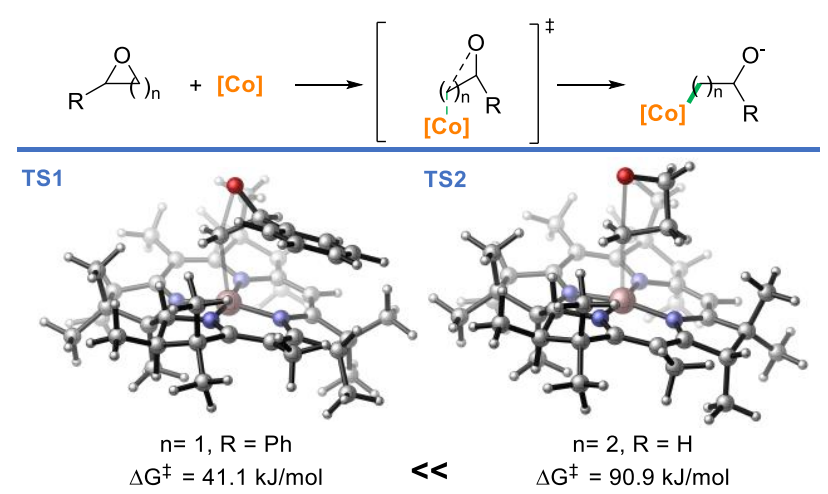

Scheme 4. The influence of acids on the Co/Ni cross-electrophile coupling of oxetane 1 with aryl iodide $\mathbf{2}^{\mathrm{a}}$

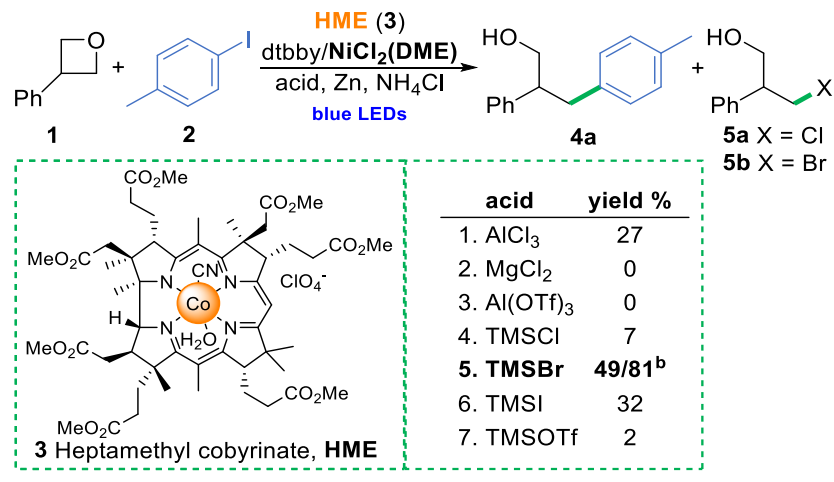


${ }^{\text {a}}$ Reaction conditions: oxetane (1, 1.5 equiv), 4-iodotoluene (2, $0.1 \mathrm{mmol}, 1$ equiv), $\mathrm{Zn}$ ( 3 equiv), $\mathrm{NH}_{4} \mathrm{Cl}(3 \mathrm{equiv}), \mathrm{HME}(\mathbf{3}, 5 \mathrm{~mol} \%)$, $\mathrm{NiCl}_{2}$ (DME) (20 mol\%), dtbby (40 mol\%), Lewis acid (2 equiv), $\mathrm{MeCN}_{\text {anh. }}(c=0.1 \mathrm{M})$, blue LEDs $(460 \mathrm{~nm}), 16 \mathrm{~h} . ;$ 1/2 1:1.5; cyields determined by GC.

In the presence of Brønsted acids (TFA, $p$ TSA), only the reductive ring-opening occurred. In contrast, some Lewis acids promoted the formation of the desired product but a notable difference in the reactivity was observed depending on the anion of the salt (see SI). DFT calculations suggest that the ring-opening of oxetanes that are activated via coordination of $\mathrm{AlCl}_{3}$, a prototypical Lewis acid, is practically barrierless indeed, in the experiment with $\mathrm{AlCl}_{3}$, product $\mathbf{4 a}$ formed in $27 \%$ yield and halohydrin $\mathbf{5 a}$ was observed as a side product, whilst the use of $\mathrm{Al}(\mathrm{OTf})_{3}$ led exclusively to the products resulting from the reductive ring opening. Therefore, we hypothesized that the halohydrin observed might act as an intermediate in our reaction. Based on further screening of halide-containing Lewis acids, $\mathrm{TMSBr}$ proved the most effective activator in promoting the model reaction. Along this line, the Jacobsen group reported enantioselective addition of TMSBr to oxetanes giving silylated bromohydrins. ${ }^{7,25}$ This work and the control experiment with TMSOTf corroborate our assumption that silylated bromohydrin $\mathbf{5 b}$ is an intermediate in this reaction.

At this point, we questioned the requirements for the Co-catalyst as Ni-catalyzed cross-electrophile coupling reactions of aryl iodides with alkyl halides are known. On the other hand, the Zultanski group has recently showed that the use of a $\mathrm{Ni} / \mathrm{Co}$ dual catalytic system eliminates complications for reaction optimizations as a Ni-catalyst activates an aryl halide while Co-catalysis induces the generation of radicals from an alkyl halide. ${ }^{26}$ Consequently, generation of reactive intermediates can be tuned separately enabling an efficient reaction. When our model reaction was performed in the presence of only the Ni-complex, we observed mainly homocoupling of aryl iodide with the product $4 \mathbf{a}$ being formed in a mere 5\% yield confirming the crucial role of the Co-catalyst in our system.

In-depth optimization studies revealed that various $\mathrm{Ni}$-complexes catalyze the cross-electrophile ring opening of oxetane 1 with aryl iodide 2 , with $\mathrm{NiCl}_{2}$ (DME) the yield significantly increased to an appreciable 84\% (Table 1, entries 3 and 4, for details see SI). Expectedly, the addition of 4,4'-di-tertbutyl-bipyridine as a ligand was required and the use of bipyridine or a ligand with diminished electron density instead decreased the yield (entry 5 and 6).

Table 1. Optimization studies of the Co/Ni cross-electrophile coupling

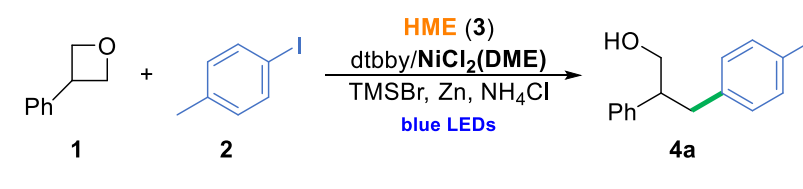

\begin{tabular}{|c|c|c|}
\hline $\begin{array}{c}\text { entr } \\
y\end{array}$ & $\begin{array}{l}\text { deviation from } \\
\text { the standard conditions }\end{array}$ & $\begin{array}{l}\text { yield } \\
(\%) 4 a^{b}\end{array}$ \\
\hline 1 & None & $84 / 80^{c}$ \\
\hline 2 & $\mathrm{Co}(\mathrm{dmgH})_{2} \mathrm{Cl}(\mathrm{py})$ instead of HME & 0 \\
\hline 3 & $\mathrm{Ni}(\mathrm{OTf})_{2}$ instead of $\mathrm{NiCl}_{2}(\mathrm{DME})$ & 76 \\
\hline 4 & $\mathrm{NiI}_{2}$ instead of $\mathrm{NiCl}_{2}(\mathrm{DME})$ & 56 \\
\hline 5 & Bpy instead of dtbbpy & 65 \\
\hline 6 & $\left(4-\mathrm{CO}_{2} \mathrm{Me}\right) \mathrm{Bpy}$ instead of dtbbpy & 25 \\
\hline 7 & HME (3) not added & 0 \\
\hline
\end{tabular}

${ }^{\mathrm{a} C}$ Conditions: oxetane (1, $\left.0.2 \mathrm{mmol}\right)$, aryl halide (2, 1.5 equiv), $\mathrm{HME} \mathrm{(3,6} \mathrm{mol \% ),} \mathrm{NiCl}_{2}(\mathrm{DME})(15 \mathrm{~mol} \%), \mathrm{Zn}(3 \mathrm{equiv}), \mathrm{NH}_{4} \mathrm{Cl}(3 \mathrm{equiv})$, dtbbpy (20 mol\%), TMSBr (2 equiv), $\mathrm{MeCN}_{\text {anh. }}(c=0.1 \mathrm{M})$, time $16 \mathrm{~h}$, blue LEDs (tape, $460 \mathrm{~nm}$ ); ${ }^{\mathrm{b}} \mathrm{GC}$ yield, ${ }^{c}$ Isolated yield, dmgH dimethylglyoxime, dtbbpy - 4,4'-di-tert-butyl-bipyridine, bpy - 2,2'-bipyridine. 
To check how the optimization process leveraged the effect of the cobalt catalyst, the model reaction was performed under the optimized conditions but with no HME (3) added (entry 7). The reaction stopped at the bromohydrin formation step (93\%) corroborating exclusive generation of alkyl radicals only in the Co-catalytic cycle.

Having identified the optimal reaction conditions, we examined the generality of the developed method (Scheme 5A). Initially, we focused our effort on 3-phenyl substituted oxetanes of type $\mathbf{1}$. Oxetanes with an electron-deficient phenyl ring yielded the product while those with an electrondonating substituent did not. In general, various substituents at the $\mathrm{C} 3$ position are well tolerated. Not only aryl and heteroaryl substituents but also protected hydroxy- and amino groups can be present at this position. Alkyl groups, on the other hand, can occupy both the $\mathrm{C} 3$ and $\mathrm{C} 2$ positions giving the corresponding products $(\mathbf{8}, \mathbf{1 0})$ in 65 and $76 \%$ yield respectively.

In regard to the aryl halide, a wide range of phenyl iodides with both electron-withdrawing and electrondonating substituents at the C-4 position are suitable starting materials (alcohols 12). In contrast, for more hindered halides, the yield slightly decreased. 2-Iodo- and 3-iodotoluen furnished desired products 17 and 18 in $60 \%$ and $63 \%$ yield, respectively. It is worth mentioning, that although vitamin $\mathrm{B}_{12}$ is a known catalyst for dehalogenation reactions, ${ }^{27-29}$ for aryl halides bearing $\mathrm{Cl}$ and $\mathrm{Br}$ this side-reaction was not observed. Electron-rich aryl halides are less reactive in this transformation, however, the synthesis of alcohol $\mathbf{1 9}$ was possible in 30\% yield. Gratifying even sensitive 1-iodo-4(trimethylsilylethynyl)-benzene furnished desired product 16, which can be further used in the silaSonogashira-Hagihara coupling. ${ }^{30,31}$

Like the described cross-coupling reaction, alkyl radicals generated from oxetanes should also engage in reactions with SOMOphiles. The model reaction of 3-phenyloxetane (1) with methyl acrylate (20) without any activator did not lead to desired product 21 (Scheme 6).

\section{Scheme 6. Co-catalyzed functionalization of oxetanes involving ring-opening}

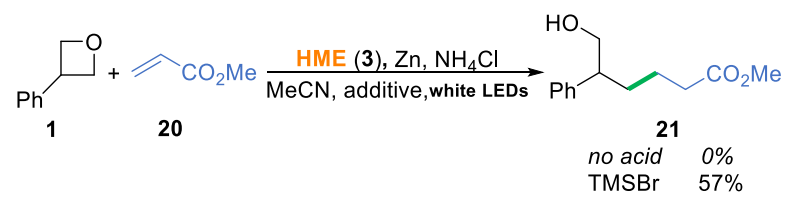

Not surprisingly, the activation with TMSBr proved also crucial in this case. The model reaction with the addition of this Lewis acid led to product 21 in 57\% yield (see also SI). Other acids tested were less effective in catalyzing the Giese-type addition (see SI). Further systematic experimental studies on the model reaction of oxetane $\mathbf{1}$ with methyl acrylate (20) in the presence of TMSBr and HME (3) identified the optimal conditions under which desired product $\mathbf{2 1}$ formed in $82 \%$ yield (Table 2).

Irradiation with blue or green light yielded the desired product, though in a diminished 57\% and 69\% yield, respectively (entries 2 and 3). Other tested cobalt complexes were less effective in catalyzing the model reaction (entries 4 and 5, see also SI, section 4.5). 
Scheme 5. Scope of the Co/Ni-catalyzed cross electrophile of oxetanes and aryl iodides and the Giese addition of oxetanes to electrophilic alkenes

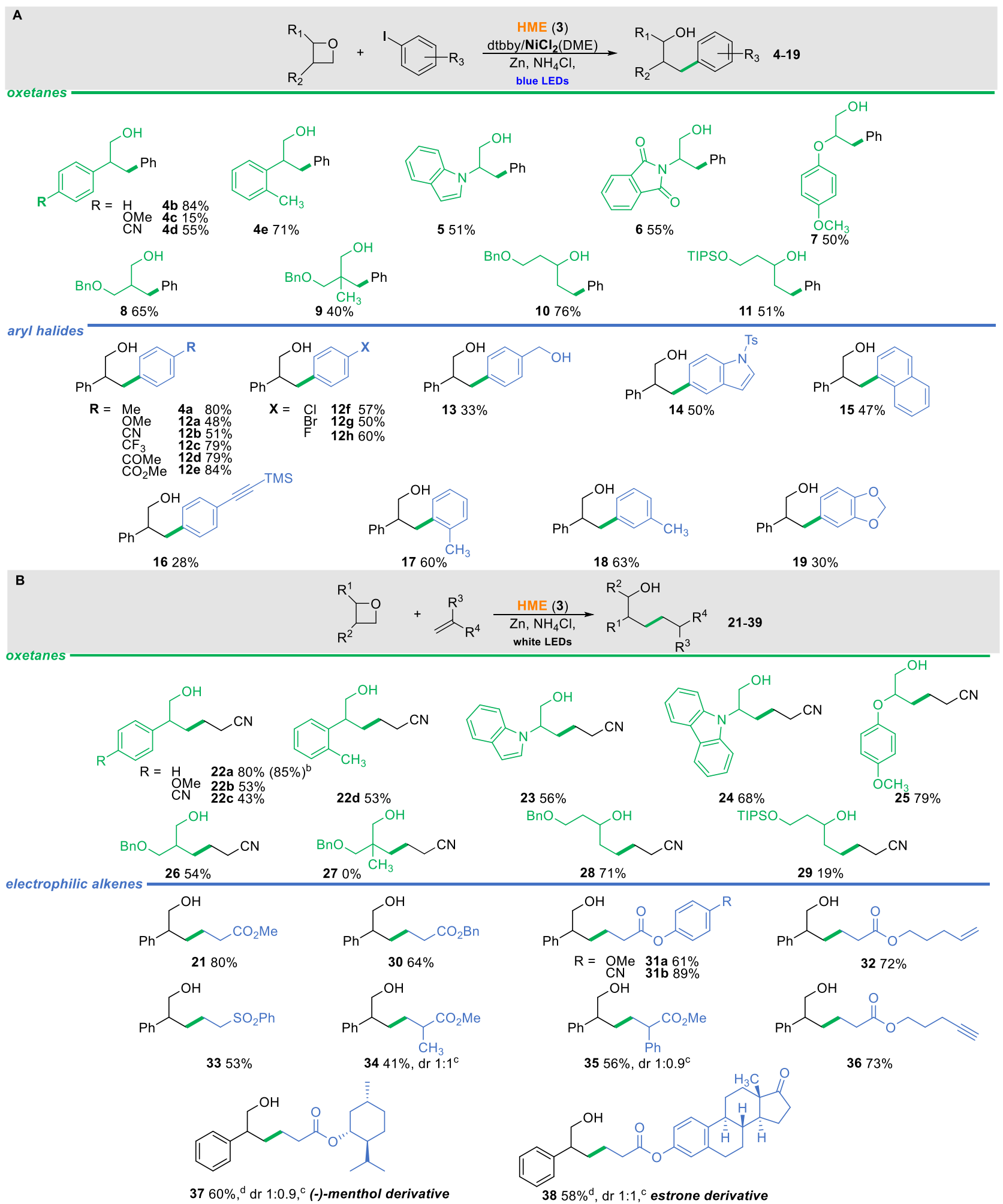

A) $)^{\mathrm{a}}$ Conditions for cross-electrophile coupling: oxetane $(0.2 \mathrm{mmol})$, aryl halide (1.5 equiv), $\left.\mathrm{HME} \mathrm{(3,6} \mathrm{mol} \%\right), \mathrm{NiCl}_{2}(\mathrm{DME})(15 \mathrm{~mol} \%)$, $\mathrm{Zn}$ (3 equiv), $\mathrm{NH}_{4} \mathrm{Cl}$ (3 equiv), dtbbpy ( $20 \mathrm{~mol} \%$ ), TMSBr (2 equiv), $\mathrm{MeCN}_{\text {anh. }}(c=0.1 \mathrm{M})$, time $16 \mathrm{~h}$, blue LEDs (tape); each reaction was quenched by treatment 2 equiv of citric acid, for $11 \mathrm{~K}_{2} \mathrm{CO}_{3}$ was used. B) ${ }^{\mathrm{a}}$ Conditions for Giese addition: oxetane ( $\left.0.2 \mathrm{mmol}\right)$, Michael acceptor

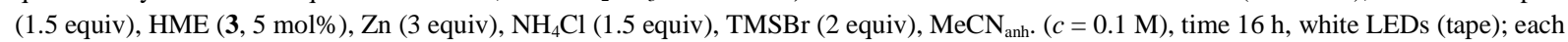
reaction was quenched by treatment 2 equiv of citric acid, ${ }^{b}$ reaction was performed on $0.1 \mathrm{mmol}$ scale. ${ }^{\mathrm{c}} \mathrm{dr}$ determined by ${ }^{13} \mathrm{C} \mathrm{NMR},{ }^{\mathrm{d}}(c=0.3$ $\mathrm{M})$. 
Table 2. Optimization studies of the Giese reaction ${ }^{a}$

\begin{tabular}{|c|c|c|}
\hline$h_{1}$ & $\begin{array}{l}\mathrm{HME}(\mathbf{3}), \mathrm{TMSBr} \text {, } \\
\qquad \begin{array}{l}\mathrm{Zn}, \mathrm{NH}_{4} \mathrm{Cl} \\
\text { white LEDs }\end{array}\end{array}$ & 29 \\
\hline $\begin{array}{c}\text { entr } \\
y\end{array}$ & $\begin{array}{l}\text { deviation from } \\
\text { the standard conditions }\end{array}$ & $\begin{array}{l}\text { yield } \\
(\%) 21^{b}\end{array}$ \\
\hline 1 & None & $82 / 80^{c}$ \\
\hline 2 & $\begin{array}{l}\text { Blue (460 nm) light instead of } \\
\text { white light }\end{array}$ & 57 \\
\hline 3 & $\begin{array}{l}\text { Green }(525 \mathrm{~nm}) \text { light instead of } \\
\text { white light }\end{array}$ & 69 \\
\hline 4 & $\begin{array}{l}\text { Co(II) phthalocyanine instead of } \\
\text { HME }\end{array}$ & 31 \\
\hline 5 & $\mathrm{Co}(\mathrm{dmgH})_{2} \mathrm{Cl}(\mathrm{py})$ instead of HME & 0 \\
\hline
\end{tabular}

${ }^{\mathrm{a}}$ Conditions: oxetane (1, $\left.0.2 \mathrm{mmol}\right)$, acrylate (20, 1.5 equiv), $\mathrm{HME}(\mathbf{3}, 5 \mathrm{~mol} \%), \mathrm{Zn}$ (3 equiv), $\mathrm{NH}_{4} \mathrm{Cl}$ (1.5 equiv), $\mathrm{TMSBr}\left(2\right.$ equiv), $\mathrm{MeCN}_{\text {anh. }}$ $\left(c=0.1 \mathrm{M}\right.$ ), time $16 \mathrm{~h}$, white LEDs (tape) (for more details see SI), ${ }^{b} \mathrm{GC}$ yield, ${ }^{c}$ Isolated yield; $\mathrm{dmgH}$ - dimethylglyoxime.

The reactivity of oxetanes in the Giese-type addition follows a similar pattern as in the crosscoupling reaction (Scheme 5B). Both EDG and EWG at the 3-phenyl substituent are well tolerated (22b, 22c, 53\% and $43 \%$, respectively) and the substitution pattern did not significantly affect the reaction yield. Both $N$-oxetanyl indole and carbazole underwent the reaction effectively giving 23 and 24 in $56 \%$ and $68 \%$ yield. Furthermore, oxetanes bearing a protected hydroxy group are well tolerated though the protecting group must be chosen carefully, as silyloxy oxetane furnished product $\mathbf{2 9}$ in low yield. C2Alkyl substituted substrates behaved similarly to oxiranes, the ring opening occurred at the less hindered site due to the steric hindrance, a feature characteristic for vitamin $\mathrm{B}_{12}$-catalyzed reactions. ${ }^{14}$ These results are however in contrast to reports from Grimme. ${ }^{11}$ Both the $\mathrm{Cp}_{2} \mathrm{TiCl}_{2}$-catalyzed reaction lead to primary alcohols as the major product. Our newly developed Co-based methodology gives access to regioisomeric products thus complementing the existing approaches.

A large array of electron-deficient olefins is well tolerated for the reaction (Scheme 5B). Acrylates

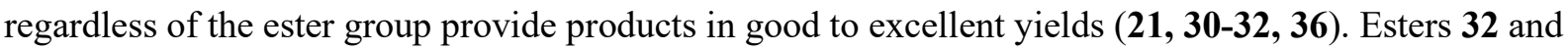
36, containing a terminal double and triple bond respectively, are worth mentioning as no reduction was observed at these ends. 1,2-Disubstituted olefins furnish products though with very low yield. However, the presence of a substituent at the $\alpha$-position to the ester group does not have a negative impact on the reaction (34 and 35). The utility of the developed method was realized when applying it to complex molecules such as an estrone derivative. Due to an issue with solubility, this reaction was performed at a lower concentration and as a result the yield of product 38 significantly increased from 28 to $58 \%$.

To gain a better understanding of the developed transformations, several mechanistic experiments were performed (Figure 1, see also section 7 in the $S I$ ). Firstly, control experiments revealed that the cobalt complex, reducing agent, and light are all crucial for the reaction of oxetanes with electrondeficient olefins to occur. Secondly, the radical nature of this process was supported by the complete shutdown of the reaction in the presence of a radical trap (TEMPO, Figure 1A). 


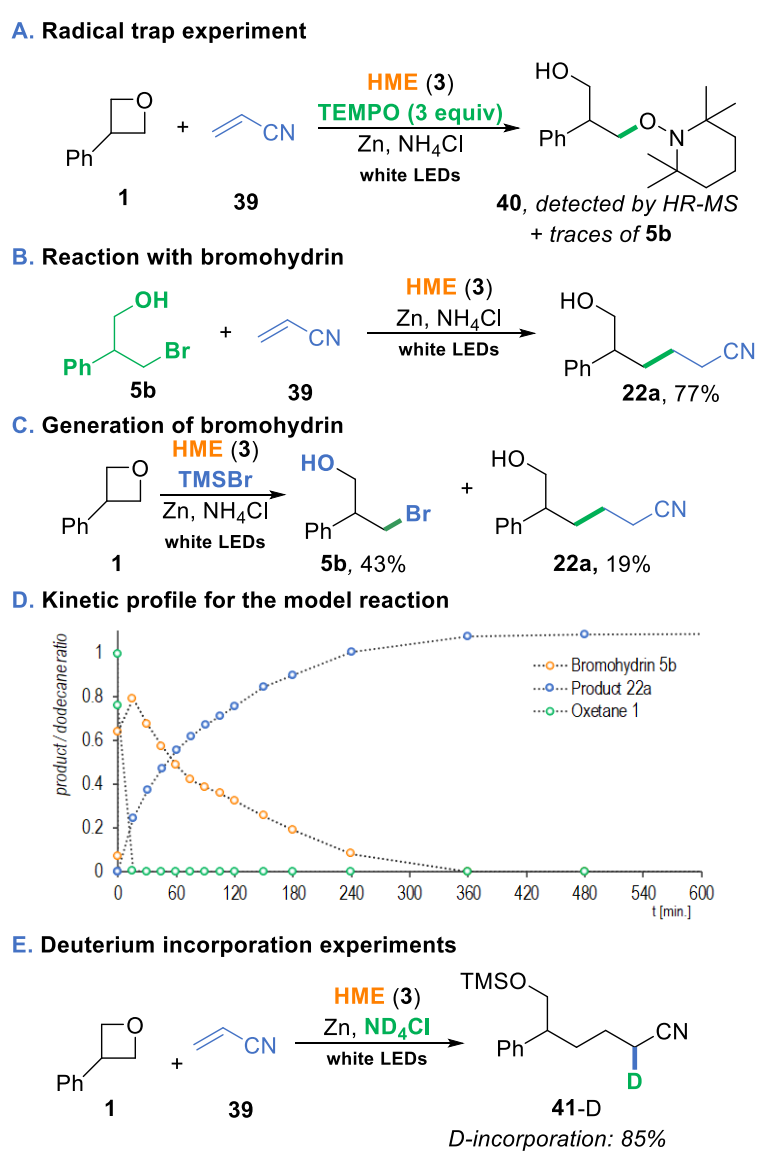

Figure 1. Mechanistic experiments

Thirdly, as already demonstrated, TMSBr plays a crucial role. Since this reagent was shown to react with oxetanes giving bromohydrins, we considered that this reaction may play a role in the generation of alkyl radicals from oxetanes as well. To examine the intermediacy of the bromohydrin, compound $\mathbf{5 b}$ was subjected to the reaction conditions (Figure 1B). Product 21 formed in 77\% yield, suggesting that a bromohydrin is indeed involved in the catalytic cycle. In the absence of the olefin, the conversion of oxetane 1 was full after 5 min (Figure 1C). In addition, even the model reaction stopped after $10 \mathrm{~min}$ afforded bromohydrin $\mathbf{5 b}(43 \%)$ as the major product with only traces of the Giese product $\mathbf{2 2} \mathbf{a}(19 \%)$. The kinetic experiments clearly show that the oxetane is fully converted into bromohydrin within first few minutes even when the olefin is present, while the product gradually forms over $10 \mathrm{~h}$ (Figure 1D). In addition, the MS analysis also shows the peak corresponding to the alkyl-cobalt complex $(\mathrm{m} / \mathrm{z}=$ $\left.1243.5671\left[\mathrm{M}+\mathrm{H}^{+}\right]\right)$. Furthermore, the model reaction performed with deuterated reagents $\left(\mathrm{ND}_{4} \mathrm{Cl}\right)$ showed deuterium-incorporation only at the $\alpha$-position to the electron withdrawing group originating from the olefin (Figure 1E). This indicates the formation of an anion at this position.

Based on our mechanistic considerations, we proposed the key steps in the developed transformations (Scheme 7). These involve the formation of bromohydrin I from oxetane that subsequently reacts with a $\mathrm{Co}$ (I) species giving alkylated cobalt complex II. The homolytic cleavage of the Co-C-bond generates alkyl radical III that either is trapped by electron-deficient olefin or enters the Ni-catalytic cycle. 
Scheme 7. Proposed a mechanism; I path: Giese-type addition, II path: Co/Ni cross coupling

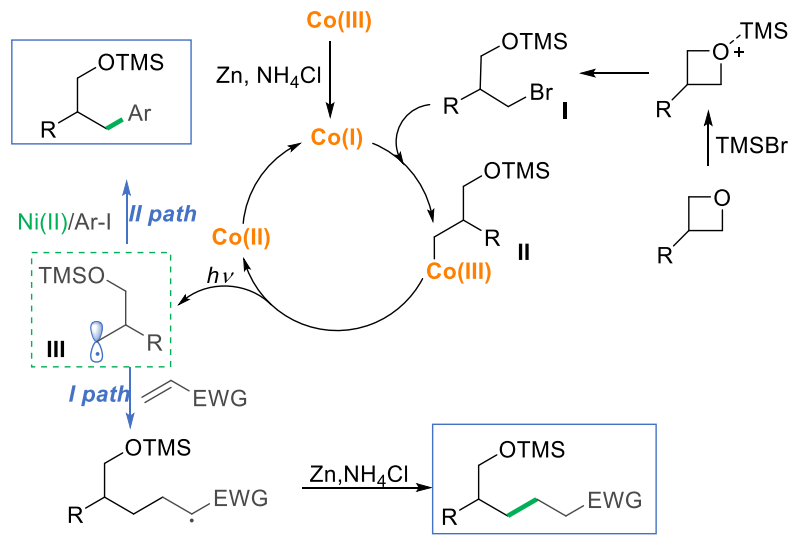

The proposed steps leading to alkyl radicals are supported by DFT calculations (Figure 2). The calculated Gibbs free energy profile for the generation of radicals from oxetane catalyzed by the Cocorrin complex is depicted in Figure 2. Firstly, oxetane undergoes exergonic ring opening with $\mathrm{TMSBr}$ $(\Delta \mathrm{G}=-51.4 \mathrm{~kJ} / \mathrm{mol})$. The resulting silyl ether of $\gamma$-bromohydrin enters a facile reaction with nucleophilic $\mathrm{Co}(\mathrm{I})$ complex through a $\mathrm{S}_{\mathrm{N}} 2$ manifold $^{32,33}$

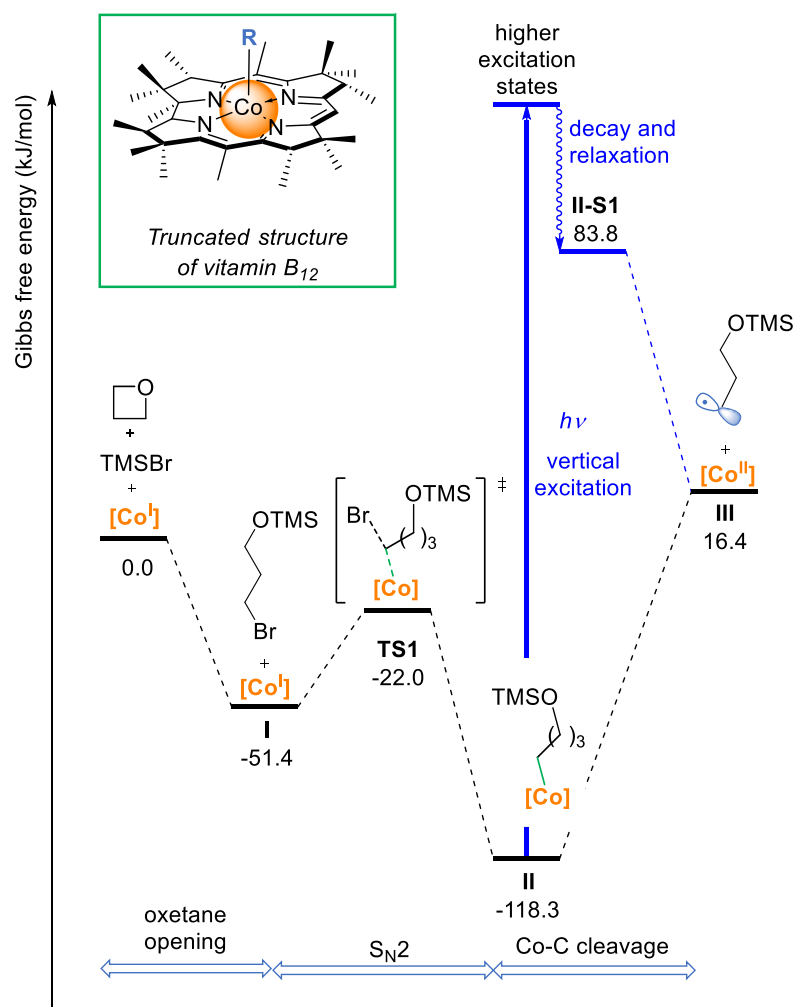

Figure 2. Calculated Gibbs free energy profile for the reaction of oxetanes with the Co(I)-corrin complex in the presence of TMSBr.

$\left(\Delta \mathrm{G}^{\ddagger}=29.4 \mathrm{~kJ} / \mathrm{mol}\right)$, giving rise to a $\mathrm{Co}(\mathrm{III})$-alkyl intermediate II, featuring a relatively weak $\mathrm{Co}-\mathrm{C}(\mathrm{sp} 3)$. Moreover, II is photoactive in visible region, which can trigger a homolytic cleavage of Co$\mathrm{C}$ bond providing alkyl radical III and the Co(II) complex. As proposed by Kozlowski, photodissociation proceeds presumably from the first electronically excited state (S1) through the generation of a singlet radical pair. ${ }^{34-36}$ 


\section{Conclusions}

Herein, we described the vitamin $\mathrm{B}_{12}$ assisted generation of $\mathrm{C}$-centered alkyl radicals from oxetanes via an alkylated cobalt complex. Subsequent, light-induced homolysis of the $\mathrm{Co}-\mathrm{C}$ bond yields radical species that engage in reactions with SOMOphiles and low-valent metal complexes. Thus, this useful C3 synthon can now be employed in various radical reactions such as Giese addition and crosselectrophile coupling. Both reactions tolerate a broad range of starting materials with different functional groups. The unique regioselectivity of the developed reaction complements the existing strategies; here the less substituted $\mathrm{C} 2$ carbon atom is functionalized. These examples suggest that Co-catalysis will enable other radical transformation of oxetanes to be developed.

Ultimately, we believe that the reported activation mode for the generation of C-centered radicals opens new opportunities in radical chemistry of oxetanes and will enable broader application of this valuable $\mathrm{C} 3$ synthon in the construction of complex molecules.

\section{ASSOCIATED CONTENT}

\section{Supporting Information}

The Supporting Information is available free of charge on the ACS Publications website at DOI: XXX. Experimental details and procedures, optimization studies, mechanistic experiments, DFT, and spectral data for all new compounds (PDF).

\section{AUTHOR INFORMATION}

\section{Corresponding Author}

Dorota Gryko - Institute of Organic Chemistry, Polish Academy of Sciences 01-224 Warsaw, Poland

* E-mail: dorota.gryko@icho.edu.pl

Wojciech Chaładaj - Institute of Organic Chemistry, Polish Academy of Sciences 01-224 Warsaw, Poland

*E-mail: wojciech.chaladaj@icho.edu.pl

\section{Author Contributions}

These authors contributed equally to this work.

W. Chaładaj performed DFT calculations.

\section{Notes}

The authors declare no competing financial interests.

\section{ACKNOWLEDGMENT}

Financial support for this work was provided by the National Science Foundation (D.G. MAESTRO UMO-2020/38/A/ST4/00185) and the Foundation for Polish Sciences (A.P. FNP TEAM POIR.04.04.00-00-4232/17-00, M.O. no. START 64.2020). Calculations have been carried out using resources provided by Wrocław Centre for Networking and Supercomputing (http://wcss.pl), grant No. 518.

\section{REFERENCES}

(1) Bull, J. A.; Croft, R. A.; Davis, O. A.; Doran, R.; Morgan, K. F. Oxetanes: Recent Advances in Synthesis, Reactivity, and Medicinal Chemistry. Chem. Rev. 2016, 116, 12150-12233. https://doi.org/10.1021/acs.chemrev.6b00274.

(2) Sandvoß, A.; Wiest, J. M. Recent Advances in Enantioselective Desymmetrizations of Prochiral Oxetanes. Chem. - A Eur. J. 2021, 27, 5871-5879. https://doi.org/10.1002/chem.202004923. 
(3) Huang, H.; Zhang, T.; Sun, J. Mild C-C Bond Formation via Lewis Acid Catalyzed Oxetane Ring Opening with Soft Carbon Nucleophiles. Angew. Chemie - Int. Ed. 2021, 60, 2668-2673. https://doi.org/10.1002/anie.202013062.

(4) Wang, C. Electrophilic Ring Opening of Small Heterocycles. Synthesis (Stuttg). 2017, 49, 53075319. https://doi.org/10.1055/s-0036-1589102.

(5) Yang, W.; Wang, Z.; Sun, J. Enantioselective Oxetane Ring Opening with Chloride: Unusual Use of Wet Molecular Sieves for the Controlled Release of HCl. Angew. Chemie - Int. Ed. 2016, 55, 6954-6958. https://doi.org/10.1002/anie.201601844.

(6) Wang, Z.; Chen, Z.; Sun, J. Catalytic Enantioselective Intermolecular Desymmetrization of 3Substituted Oxetanes. Angew. Chemie Int. Ed. 2013, 52, 6685-6688. https://doi.org/10.1002/anie.201300188.

(7) Strassfeld, D. A.; Wickens, Z. K.; Picazo, E.; Jacobsen, E. N. Highly Enantioselective, Hydrogen-Bond-Donor Catalyzed Additions to Oxetanes. J. Am. Chem. Soc. 2020, 142, 91759180. https://doi.org/10.1021/jacs.0c03991.

(8) Jin, J.; MacMillan, D. W. C. Direct $\alpha$-Arylation of Ethers through the Combination of Photoredox-Mediated C-H Functionalization and the Minisci Reaction. Angew. Chemie - Int. Ed. 2015, 54, 1565-1569. https://doi.org/10.1002/anie.201410432.

(9) Ravelli, D.; Zoccolillo, M.; Mella, M.; Fagnoni, M. Photocatalytic Synthesis of Oxetane Derivatives by Selective C-H Activation. Adv. Synth. Catal. 2014, 356, 2781-2786. https://doi.org/10.1002/adsc.201400027.

(10) Gansäuer, A.; Ndene, N.; Lauterbach, T.; Justicia, J.; Winkler, I.; Mück-Lichtenfeld, C.; Grimme, S. Titanocene Catalyzed Opening of Oxetanes. Tetrahedron 2008, 64, 11839-11845. https://doi.org/10.1016/j.tet.2008.08.107.

(11) Takekoshi, N.; Miyashita, K.; Shoji, N.; Okamoto, S. Generation of a Low-Valent Titanium Species from Titanatrane and Its Catalytic Reactions: Radical Ring Opening of Oxetanes. Adv. Synth. Catal. 2013, 355, 2151-2157. https://doi.org/10.1002/adsc.201300368.

(12) Sugiyama, Y. K.; Heigozono, S.; Okamoto, S. Iron-Catalyzed Reductive Magnesiation of Oxetanes to Generate (3-Oxidopropyl)Magnesium Reagents. Org. Lett. 2014, 16, 6278-6281. https://doi.org/10.1021/ol503191w.

(13) Ociepa, M.; Wierzba, A. J.; Turkowska, J.; Gryko, D. Polarity-Reversal Strategy for the Functionalization of Electrophilic Strained Molecules via Light-Driven Cobalt Catalysis. J. Am. Chem. Soc. 2020, 142, 5355-5361. https://doi.org/10.1021/jacs.0c00245.

(14) Potrząsaj, A.; Musiejuk, M.; Chaładaj, W.; Giedyk, M.; Gryko, D. Cobalt Catalyst Determines Regioselectivity in Ring Opening of Epoxides with Aryl Halides. J. Am. Chem. Soc. 2021, 143, 9368-9376. https://doi.org/10.1021/jacs.1c00659.

(15) Komeyama, K.; Michiyuki, T.; Osaka, I. Nickel/Cobalt-Catalyzed C(Sp 3 )-C(Sp 3 ) CrossCoupling of Alkyl Halides with Alkyl Tosylates. ACS Catal. 2019, 9, 9285-9291. https://doi.org/10.1021/acscatal.9b03352.

(16) Komeyama, K.; Ohata, R.; Kiguchi, S.; Osaka, I. Highly Nucleophilic Vitamin B 12 -Assisted Nickel-Catalysed Reductive Coupling of Aryl Halides and Non-Activated Alkyl Tosylates. Chem. Commun. 2017, 53, 6401-6404. https://doi.org/10.1039/C7CC01932G.

(17) Komeyama, K.; Tsunemitsu, R.; Michiyuki, T.; Yoshida, H.; Osaka, I. Ni/Co-Catalyzed Homo$\begin{array}{llllll}\text { Coupling of Alkyl Tosylates. Molecules 2019, } & \text { 24, } & 1458 .\end{array}$ https://doi.org/10.3390/molecules24081458.

(18) Shevick, S. L.; Obradors, C.; Shenvi, R. A. Mechanistic Interrogation of Co/Ni-Dual Catalyzed Hydroarylation. J. Am. Chem. Soc. 2018, 140, 12056-12068. https://doi.org/10.1021/jacs.8b06458.

(19) Green, S. A.; Matos, J. L. M.; Yagi, A.; Shenvi, R. A. Branch-Selective Hydroarylation: 
Iodoarene-Olefin Cross-Coupling. J. Am. Chem. Soc. 2016, 138, 12779-12782. https://doi.org/10.1021/jacs.6b08507.

(20) Ackerman, L. K. G.; Anka-Lufford, L. L.; Naodovic, M.; Weix, D. J. Cobalt Co-Catalysis for Cross-Electrophile Coupling: Diarylmethanes from Benzyl Mesylates and Aryl Halides. Chem. Sci. 2015, 6, 1115-1119. https://doi.org/10.1039/c4sc03106g.

(21) Hofstra, J. L.; Cherney, A. H.; Ordner, C. M.; Reisman, S. E. Synthesis of Enantioenriched Allylic Silanes via Nickel-Catalyzed Reductive Cross-Coupling. J. Am. Chem. Soc. 2018, 140, 139-142. https://doi.org/10.1021/jacs.7b11707.

(22) Frisch, M. J.; Trucks, G. W.; Schlegel, H. B.; Scuseria, G. E.; Robb, M. A.; Cheeseman, J. R.; Scalmani, G.; Barone, V.; Petersson, G. A.; Nakatsuji, H.; Li, X.; Caricato, M.; Marenich, A. V.; Bloino, J.; Janesko, B. G.; Gomperts, R.; Mennucci, B.; Hratchian, H. P.; Ortiz, J. V.; Izmaylov, A. F.; Sonnenberg, J. L.; Williams-Young, D.; Ding, F.; Lipparini, F.; Egidi, F.; Goings, J.; Peng, B.; Petrone, A.; Henderson, T.; Ranasinghe, D.; Zakrzewski, V. G.; Gao, J.; Rega, N.; Zheng, G.; Liang, W.; Hada, M.; Ehara, M.; Toyota, K.; Fukuda, R.; Hasegawa, J.; Ishida, M.; Nakajima, T.; Honda, Y.; Kitao, O.; Nakai, H.; Vreven, T.; Throssell, K.; Montgomery, J. A., Jr.; Peralta, J. E.; Ogliaro, F.; Bearpark, M. J.; Heyd, J. J.; Brothers, E. N.; Kudin, K. N.; Staroverov, V. N.; Keith, T. A.; Kobayashi, R.; Normand, J.; Raghavachari, K.; Rendell, A. P.; Burant, J. C.; Iyengar, S. S.; Tomasi, J.; Cossi, M.; Millam, J. M.; Klene, M.; Adamo, C.; Cammi, R.; Ochterski, J. W.; Martin, R. L.; Morokuma, K.; Farkas, O.; Foresman, J. B.; Fox, D. J. Gaussian 16, Revision B.01; Gaussian, Inc.: Wallingford, CT, 2016

(23) Grimme, S.; Antony, J.; Ehrlich, S.; Krieg, H. A Consistent and Accurate Ab Initio Parametrization of Density Functional Dispersion Correction (DFT-D) for the 94 Elements HPu. J. Chem. Phys. 2010, 132. https://doi.org/10.1063/1.3382344.

(24) Marenich, A. V.; Cramer, C. J.; Truhlar, D. G. Universal Solvation Model Based on Solute Electron Density and on a Continuum Model of the Solvent Defined by the Bulk Dielectric Constant and Atomic Surface Tensions. J. Phys. Chem. B 2009, 113, 6378-6396. https://doi.org/10.1021/jp810292n.

(25) Strassfeld, D. A.; Algera, R. F.; Wickens, Z. K.; Jacobsen, E. N. A Case Study in Catalyst Generality: Simultaneous, Highly-Enantioselective Brønsted- And Lewis-Acid Mechanisms in Hydrogen-Bond-Donor Catalyzed Oxetane Openings. J. Am. Chem. Soc. 2021, 143, 9585-9594. https://doi.org/10.1021/jacs.1c03992.

(26) Charboneau, D. J.; Barth, E. L.; Hazari, N.; Uehling, M. R.; Zultanski, S. L. A Widely Applicable Dual Catalytic System for Cross-Electrophile Coupling Enabled by Mechanistic Studies. ACS Catal. 2020, 10, 12642-12656. https://doi.org/10.1021/acscatal.0c03237.

(27) Giedyk, M.; Goliszewska, K.; Gryko, D. Vitamin B 12 Catalysed Reactions. Chem. Soc. Rev. 2015, 44, 3391-3404. https://doi.org/10.1039/c5cs00165j.

(28) Hisaeda, Y.; Tahara, K.; Shimakoshi, H.; Masuko, T. Bioinspired Catalytic Reactions with Vitamin $\mathrm{B}_{12}$ Derivative and Photosensitizers. Pure Appl. Chem. 2013, 85, 1415-1426. https://doi.org/10.1351/pac-con-12-10-05.

(29) Anai, Y.; Shichijo, K.; Fujitsuka, M.; Hisaeda, Y.; Shimakoshi, H. Synthesis of a B 12 -BODIPY Dyad for $\mathrm{B}_{12}$-Inspired Photochemical Transformations of a Trichloromethylated Organic Compound. Chem. Commun. 2020, 56, 11945-11948. https://doi.org/10.1039/d0cc04274a.

(30) Ihara, H.; Ueda, A.; Suginome, M. Ruthenium-Catalyzed C-H Silylation of Methylboronic Acid Using a Removable $\alpha$-Directing Modifier on the Boron Atom. Chem. Lett. 2011, 40, 972-974. https://doi.org/10.1246/cl.2011.972.

(31) Buendia, J.; Darses, B.; Dauban, P. Tandem Catalytic C(Sp3)-HAmination/Sila-SonogashiraHagihara Coupling Reactions with Iodine Reagents. Angew. Chemie - Int. Ed. 2015, 54, 5697 5701. https://doi.org/10.1002/anie.201412364. 
(32) Zhou, D. -L; Walder, P.; Scheffold, R.; Walder, L. SN2 or Electron Transfer?? A New Technique Discriminates the Mechanisms of Oxidative Addition of Alkyl Halides to Corrinato- and Porphyrinatocobalt(I). Helv. Chim. Acta 1992, 75, 995-1011. https://doi.org/10.1002/hlca.19920750403.

(33) Ghosh, A. P.; Lodowski, P.; Chmielowska, A.; Jaworska, M.; Kozlowski, P. M. Elucidating the Mechanism of Cob(I)Alamin Mediated Methylation Reactions by Alkyl Halides: SN2 or Radical Mechanism? J. Catal. 2019, 376, 32-43. https://doi.org/10.1016/j.jcat.2019.06.036.

(34) Kozlowski, P. M.; Garabato, B. D.; Lodowski, P.; Jaworska, M. Photolytic Properties of Cobalamins: A Theoretical Perspective. Dalt. Trans. 2016, 45, 4457-4470. https://doi.org/10.1039/c5dt04286k.

(35) Garabato, B. D.; Lodowski, P.; Jaworska, M.; Kozlowski, P. M. Mechanism of Co-C Photodissociation in Adenosylcobalamin. Phys. Chem. Chem. Phys. 2016, 18, 19070-19082. https://doi.org/10.1039/c6cp02136k.

(36) Lodowski, P.; Jaworska, M.; Garabato, B. D.; Kozlowski, P. M. Mechanism of Co-C Bond Photolysis in Methylcobalamin: Influence of Axial Base. J. Phys. Chem. A 2015, 119, 39133928. https://doi.org/10.1021/jp5120674. 